\title{
Wussten Sie schon...
}

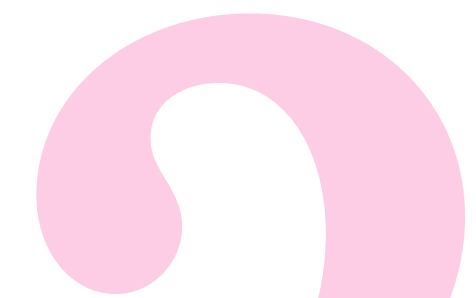

... dass Gehen nicht nur gut ist für das kardiovaskuläre System, sondern auch für das Gehirn?

Ein Forscherteam der University of Illinois verglich die Auswirkung auf gewisse Hirnfunktionen 6 Monate nach einem Gehtraining im Vergleich zu Stretching oder tonischen Übungen bei älteren Menschen [Nature 1999; 400:418-419]. Die Gehgruppe schnitt signifikant besser ab bei den kognitiven Tests. Am grössten war die Auswirkung des Gehens auf Prozessdenken, wie Planen und Arbeitsgedächtnis. Man nimmt an, dass diese Funktionen durch den frontalen und präfrontalen Kortex kontrolliert werden.

\section{Kommentar}

Bemerkenswert ist die selektive Wirkung dieses aeroben Trainings und die Beobachtung, dass bereits eine bescheidene Verbesserung der aeroben Fitness (Verbesserung des maximalen $\mathrm{O}_{2}$-Verbrauchs um nur 5\%) zu den beobachteten signifikanten Veränderungen führte.

m.k.h.
... dass man Kaugummikauen als signifikanten Beitrag zur Kontrolle des Körpergewichts ansehen kann?

Die Autoren aus der Mayo-Klinik [N Engl J Med 1999;341: 2100] untersuchten die Energieabgabe in einem klimatisierten dunklen geräuschisolierten Labor mittels indirekter Kalorimetrie. Bei 7 nicht obesen Probanden wurde der Energieverbrauch vor und unmittelbar nach 12-minütigem Kaugummikauen (Kaufrequenz mittels Metronoms $100 \mathrm{~Hz}$ - dies sei die übliche Kaufrequenz an der Mayo-Klinik!). Der durchschnittliche Energieverbrauch ( \pm SD) stieg bei allen Probanden von $58 \pm 11 \mathrm{kcal} / \mathrm{h}$ vor dem Kauen auf $70 \pm 14 \mathrm{kcal} / \mathrm{h}$ nach dem Kauen ( $p<0,001)$. Der Energieverbrauch stieg somit um $11 \pm 3 \mathrm{kcal} / \mathrm{h}(+19 \%)$. Im Vergleich dazu stiegen die Werte bei den gleichen Probanden um 11\% beim Stehen und 106\% beim Gehen. Die Autoren kommen zum Schluss, dass Kaugummikauen ausreichend exotherm sei, um allein dadurch einen jährlichen Gewichtsverlust von mindestens $5 \mathrm{~kg}$ zu ermöglichen. Sie empfehlen deshalb das Kauen von kalorienfreiem Kaugummi während des Tages bei Übergewichtigen.

\section{Kommentar}

Es ist keine Überraschung, dass diese Untersuchung aus den USA kommt. In Europa hat man sicher etwas Mühe mit der Vorstellung von permanent (100 $\mathrm{mal} / \mathrm{min}$ ) kauenden Menschen. Bei Kühen, bei denen man den Anblick eher gewohnt ist, wird der Energieverbrauch durch Wiederkäuen übrigens um 20\% erhöht.

$$
\text { m.k.h. }
$$

... dass die niedrige Östrogen- $\alpha$ Expression in normalem Brustgewebe für die niedrige Mammakarzinominzidenz in Japan verantwortlich gemacht werden könnte?

Die Autoren [Lancet 1999;354: 1787-1788] verglichen den Östrogen- $\alpha$-Rezeptor-Gehalt immunhistochemisch in lobulärem sowie intralobulär duktalem Gewebe bei 74 weissen Australierinnen mit demjenigen von 52 Japanerinnen. Bei den Australierinnen stieg der Rezeptorgehalt von $12 \%$ bei den unter 50 jährigen auf $17 \%$ bei den über 50 jährigen an. Im Vergleich dazu waren die Werte bei den Japanerinnen mit 9\% (keine Variation mit dem Alter) deutlich niedriger. Die Autoren stellen die Hypothese auf, dass die Expression der Östrogenrezeptoren in normalem Brustgewebe mit erhöhtem Mammakarzinomrisiko einhergehe. Diese 
Ergebnisse wären auch eine Erklärung dafür, dass international gesehen eine schlechte Übereinstimmung besteht zwischen Mammakarzinominzidenz und Östrogenkonzentrationen. Der Grund für die unterschiedliche Östrogen- $\alpha$-Rezeptor-Expression könnte ein diätetischer sein (z.B. Phytoöstrogene in Sojaprodukten) oder durch eine höhere Down-Regulierung der Östrogenrezeptoren durch durchschnittlich höhere Östrogenkonzentrationen bei schwangeren Asiatinnen im Vergleich zu Weissen.

m.k.h.

\section{... dass die Versorgung onkologischer Patientinnen mit supportiven Massnahmen deutlich verbesserungsfähig ist?}

Nach einer Untersuchung in England [BMJ 1999;319:1520] sind jährlich etwa 33000 Patientinnen schmerztherapeutisch nicht optimal versorgt, 27500 würden von einer Gabe von Bisphosphonaten bei Knochenmetastasen und weitere 10000 von der Gabe von Wachstumsfaktoren (Leukozyten, Stimulation und Erythropoetin) profitieren. Die Mehrkosten für eine optimale supportive Therapie werden auf GBP 93 Mio pro Jahr geschätzt, die aber durch eine geringere Morbidität und weniger Spitaleinweisungen teilweise kompensiert werden könnten.
Kommentar

Die Situation in England ist durch die restriktive Politik des National Health Service unzweifelhaft gravierender als auf dem europäischen Festland. Dennoch erleben auch wir bei stationären Einweisungen immer wieder, dass die Möglichkeiten der supportiven Therapie bei Patientinnen mit Krebserkrankungen - insbesondere im fortgeschrittenen Stadium nicht hinreichend ausgeschöpft werden. Gründe hierfür sind nach unserer Erfahrung nicht nur die relativ hohen Kosten für die Medikamente, sondern teilweise auch die mangelnde onkologische Erfahrung der betreuenden Ärzte.

h.p.s.
... dass der Versuch, bei extremer kindlicher Unreife die Geburt des 2. Kindes einer Mehrlingsschwangerschaft zeitlich hinauszuzögern, durchaus sinnvoll sein kann?

In einer retrospektiven Analyse wurde über 80 Mehrlingsgeburten berichtet, bei denen nach der Geburt eines Kindes versucht wurde, die verbliebene Schwangerschaft weiter zu erhalten [Acta Obstet Gynecol Scand 1999;78:710-715]. In 65 Fällen gelang dies nicht. Bei 15 Fällen jedoch (davon 9 aus einem Invitro-Fertilisationsprogramm), unter denen sich 10-mal Zwillinge und 5-mal Drillinge befanden, konnte ein Zeitgewinn erzielt werden. Dieser betrug im Mittel 12 Tage. Die mittlere Gestationsdauer des 2. bzw. 2. und 3. Kindes betrug bei der Geburt beinahe 28 Wochen. Das Ergebnis war nicht davon abhängig, ob vorzeitige Wehen, vorzeitiger Blasensprung oder auch eine vaginale Blutung die Ursachen für die Frühgeburtlichkeit waren. In Bezug auf die neonatale Entwicklung gab es dann keinen Unterschied zwischen dem Erstgeborenen und dem verzögert geborenen Kind, wenn beide Kinder mehr als 28 Schwangerschaftswochen Gestationsalter aufwiesen. Von den verzögert geborenen Kindern überlebten 8,6 davon haben sich normal entwickelt. 
Kommentar

Was auf den ersten Blick vielleicht halbwegs erfolgreich aussehen könnte, entpuppt sich bei genauerem Hinsehen eher als reine Verzweiflungstat. Nimmt man nämlich im Vergleich zur nichtaufhaltbaren Geburt Überleben und handicapfreies Überleben als Erfolgskriterien, statt sich nur den zwar gut messbaren, aber wenig aussagekräftigen zeitlichen Zugewinn zu fokussieren, dann hat sich das Ganze nämlich nur für ein einziges Kind in dieser Studie gelohnt, bei welchem die 1. Geburt mit 21 Schwangerschaftswochen erfolgte und die des 2. Zwillings 8 Wochen später mit $1768 \mathrm{~g}$. Dieses Kind überlebte im Gegensatz zum ersten handicapfrei. Die Frage, ob Aufwand und Ertrag für eine solche Situation in einem vernünftigen Verhältnis stehen, wird dadurch relativiert, dass sowohl diese Studie wie andere kleinere Serien zeigen, dass sich das mütterliche Risiko beim Weiterführen der Schwangerschaft, bedingt natürlich durch die Chorioamnionitis bei entsprechender Überwachung, so kalkulieren lässt, dass es nicht zu fatalen Folgen kommt. Es gilt auch zu bedenken, dass solche Strategien eher dann ins Auge gefasst werden, wenn es um Sterilitätspatientinnen geht, was auch in dieser Studie häufiger der Fall war.
Wenn schon ein solches Vorgehen: Dann mit Cerclage? Darauf gibt die Literatur keine befriedigende Antwort. Folgt man einem speziellen Kommentar [Obstet Gynecol Surv 2000;55:208], dann scheint es durchaus sinnvoll zu sein, diese durchzuführen.

Eines sollte man bei aller medizinischen Abwägung aber nicht übersehen: Die Eltern sollten in eine solche Vorgehensweise eingebunden sein, möglichst vor der Geburt des ersten Mehrlings. Gerade dann nämlich, wenn die Frühgeburt sich vor jeglicher Lebensfähigkeit ereignet hat, wäre es tragisch, sich ohne Einwilligung auf ein so riskantes Manöver eingelassen zu haben, wenn es dann wenig später zur 2. Geburt kommt und dieses Mal ein Kind knapp an der Lebensgrenze mit multiplen Handicaps überlebt.

b.s.

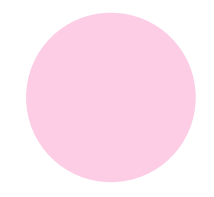

Kernaussagen

- Sorgfältige Überwachung bezüglich Chorioamnionitis

- Cerclage möglich

- Blutungen, Blasensprung, vorzeitige Wehen, keine Kontraindikationen

- Antibiotika grosszügig

- Eltern sollten eingewilligt haben 
... dass die Beinvenen mit zunehmender Schwangerschaftsdauer an Weite zunehmen?

In einer kleinen Gruppe von jeweils 43 Frauen wurde der Einfluss der Schwangerschaft auf Patientinnen mit bereits vorbestehender Varikose [Eur J Vasc Endovasc Surg 1999;18:294] und bei solchen ohne diese Probleme [Br J Obstet Gynaecol 1999; 106:597] mit der Duplexsonografie untersucht. Es zeigte sich, dass es in beiden Gruppen mit fortschreitender Schwangerschaft und einem Maximum zwischen den 37. und 40. Schwangerschaftswochen zu einer zunehmenden Dilatation des Venensystems kam. Die Ausgangswerte waren 6 Wochen nach der Schwangerschaft wieder erreicht. Bei keiner Frau wurde eine neu auftretende Klappenruptur beobachtet. Bei den Frauen mit Varikose nahm auch der Reflux zu; bei denen, welche keine Varikose hatten, zeigte sich keinerlei Reflux.
Kommentar

Obwohl ein allä̈gliches Problem in der Geburtshilfe, ist die Entwicklung von Krampfadern dennoch ein etwas vernachlässigtes Thema. Immerhin zeigen die beiden hier vorgestellten Studien, dass erweiterte Venen während der Gravidität und auch die Zunahme von Krampfadern selbst wieder auf den Stand zurückgehen, auf dem sie vor der Schwangerschaft waren; es bestätigt sich also das, was wir den Frauen sowieso mitgeteilt hätten. Unklar bleibt, wie es zur Entstehung von Krampfadern während der Gravidität kommt, welche mit einer Inzidenz von bis zu 28\% angegeben wird. Dies setzt ja die Ruptur der Venenklappen voraus, was aber in dieser Studie nicht beobachtet werden konnte. Nicht aussagekräftige Fallzahlen oder ein andererbisher unbekannter - Mechanismus, das ist die Frage. Bis dies geklärt ist, sollten wir aber die Frauen zum Tragen von Kompressionsstrümpfen motivieren. Diese haben erwiesenermassen einen günstigen Einfluss auf die Venenerweiterung und können im Einzelfall vielleicht eine Klappenruptur und damit den Beginn einer Varikose verhindern.
... dass bei über 70-jährigen Frauen die DehydroepiandrosteronSubstitution günstige Effekte auf den Knochenabbau, die Haut und auch auf die Libido hat, nicht aber bei gleichaltrigen Männern?

In einer doppelblinden und plazebokontrollierten Studie wurden 280 Männern und Frauen $50 \mathrm{mg}$ DHEA oder Plazebo über ein Jahr gegeben. Behandlungsziel war es, eine Serumkonzentration von DHEA entsprechend der Altersstufe $<50$ Jahre wieder herzustellen. Günstige Effekte auf Knochenabbau, Haut und Libido zeigten sich allerdings nur bei den Frauen [PNAS 2000;97:4279].

\section{Kommentar}

Eine gute Nachricht für die Frauen, eine schlechte für die Industrie. Diese hätte auch gerne einmal den ständig zunehmenden Markt der älteren Männer mit einer Substanz bedient, welche dem Traum vom Jungbrunnen ein wenig näher kommt.

b.s. 